\title{
Anamorelin provides benefit to patients with cachexia
}

Cachexia is a common occurrence in patients with advanced-stage cancer, with no specific treatment approaches currently approved. Now, data from a phase II study involving Japanese patients with unresectable stage III/IV non-small-cell lung cancer confirm the efficacy of the ghrelin receptor agonist anamorelin in this population.

A total of 174 patients were randomly assigned to receive either daily oral anamorelin or placebo for a total of 12 weeks, with a primary outcome measure of lean body mass (LBM). Patients receiving anamorelin had a significant improvement in LBM relative to that of patients in the placebo group of $1.56 \mathrm{~kg}(P<0.0001)$. Improvements in several secondary outcome measures, including total body mass, fat mass and quality-of-life-related outcomes, such as appetite and enjoyment of food, were also reported. No significant differences in the risk of adverse events were observed between groups. Notably, despite improving the primary outcome measure, no significant improvements were observed in measures of motor function, such as handgrip strength or 6-minute walk distance. These findings further demonstrate that cachexia is a multifactorial, chronic condition that cannot simply be reversed through improvement of appetite and/or food intake over a 12-week period. Similarly, outcomes of the prior ROMANA 3 trial revealed no improvement in motor function after 24 weeks of treatment. These findings confirm the conclusions of the previous ROMANA trials and also demonstrate that anamorelin is effective in patients with a substantially lower mean body weight than that of patients in the ROMANA trials. Anamorelin is unlikely to entirely cure patients with cachexia, although the regulatory approval and subsequent use of this agent could improve the quality of life of many patients with this syndrome.

Peter Sidaway

ORIGINAL ARTICLE Katakami, N. et al. Anamorelin (ONO-7643) for the treatment of patients with non-small cell lung cancer and cachexia: results from a randomized, double-blind, placebocontrolled, multicenter study of Japanese patients (ONO7643-04). Cancer http://dx.doi.org/10.1002/cncr.31128 (2017) 